\title{
Mireille CALLE-GRUBer, Les comptes du temps. L'archive Claude Simon. Carnets de Tante Mie
}

\section{Stefano Genetti}

\section{(2) OpenEdition}

\section{Journals}

\section{Edizione digitale}

URL: https://journals.openedition.org/studifrancesi/45493

DOI: 10.4000/studifrancesi.45493

ISSN: 2421-5856

\section{Editore}

Rosenberg \& Sellier

\section{Edizione cartacea}

Data di pubblicazione: 1 août 2021

Paginazione: 405-406

ISSN: 0039-2944

Notizia bibliografica digitale

Stefano Genetti, «Mirelle Calle-Gruber, Les comptes du temps. L'archive Claude Simon. Carnets de Tante Mie», Studi Francesi [Online], 194 (LXV | II) | 2021, online dal 01 septembre 2021, consultato il 15 octobre 2022. URL: http://journals.openedition.org/studifrancesi/45493 ; DOI: https://doi.org/10.4000/ studifrancesi.45493

Questo documento è stato generato automaticamente il 15 octobre 2022

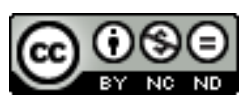

Creative Commons - Attribuzione - Non commerciale - Non opere derivate 4.0 Internazionale - CC BY NC-ND 4.0

https://creativecommons.org/licenses/by-nc-nd/4.0/ 


\title{
MiReILle CALLE-GRUBER, Les comptes $d u$ temps. L'archive Claude Simon. Carnets de Tante Mie
}

\author{
Stefano Genetti
}

\section{NOTIZIA}

Mireille Calle-Gruber, Les comptes du temps. L'archive Claude Simon. Carnets de Tante Mie, préface de Pascal Quignard, Paris, HDiffusion, 2020, 325 pp.

1 Sorella del padre di Claude Simon, ucciso in guerra nel 1914, Artémise diventa una madre sostitutiva per il nipote, che perde la sua nel 1925. Rifugiatasi a Perpignan nel giugno del Quaranta, Tante Mie muore il 25 maggio 1955 all'età di ottantacinque anni. Tra le pagine di diario dello scrittore (25-31 maggio 1955) e gli altri documenti riprodotti in calce al presente volume, in una lettera del 1960, l'autore di L'herbe (1958) si confida: «j'ai voulu écrire un livre sur cette vie admirable faite toute entière d'amour, de générosité et d'abnégation. Sa mort a été pour moi un coup très dur.... Après d'autres» (p. 300). Al romanzo si affianca la pièce teatrale La séparation, allestita nel Sessantatré ma edita, a cura della stessa Mireille Calle-Gruber, solo nel 2019. Se in appendice figura anche la fotografia di gruppo in giardino risalente alla fine dell'Ottocento, intessuta nel testo di L'herbe e riprodotta sulla copertina dell'edizione tascabile, a essere qui pubblicati sono i policromi quaderni contabili di Artémise Simon, «le fac-similé de ces dépôts qui à défaut de faire une histoire font une vie» (p. 13), scrive M. Calle-Gruber, sottolineando come alla redazione di L'herbe risalga l'adozione del sistema di colori associati a personaggi e motivi ricorrenti su cui Claude Simon basa un metodo compositivo svincolato da principi logici e cronologici.

Oltre a inquadrare in una prospettiva biografica i quattro carnets de comptes che, riprodotti integralmente o per estratti, vanno dal Ventidue al Cinquantacinque e a descriverli nella loro materialità, in «Le Livre des jours ou L'archive littéraire de Claude 
Simon» (pp. 9-35), la curatrice traccia gli itinerari di lettura che, al di là dell'industriosa aridità apparente, in filigrana si delineano tra le pagine di questo diario cifrato dal 1922 al 1955, facendone un "poema alla durata" (p. 11) dove «l'infime et l'infini» (p. 13) si intrecciano. Nel decriptare i quaderni, mostra infatti come vi si inscrivano in forma intermittente e rappresa, raffreddata, gesti e gesta, episodi domestici e ritmi stagionali, drammi privati e collettivi: la guerra, l'esodo, l'occupazione. Le somme in entrata e le voci di spesa sono le infinitesimali reliquie della «modeste, digne et exemplaire histoire de celles qui n'ont pas d'histoire et que l'Histoire du monde soudain ravage brutalement»: tracce dell'immemorabile cui corrisponde «l'élémentaire, l'involontaire, incoercible vivant qui est comme la pousse de l'herbe qu'on ne voit pas pousser» (p. 10), osserva M. Calle-Gruber riprendendo la frase di Pasternak posta in epigrafe a L'herbe per poi svilupparla in termini vegetativi, germinativi e di disseminazione.

3 È dunque questo il lascito di «menus événements (et même pas événements: faits, incidents - et même pas incidents: le quotidien, le tout-venant - et même pas menus: minuscules, insignifiants) ressurgissant hors du temps, de l'aboli» (L'herbe, Minuit «double», 2015, p. 99) che la scrittura di Claude Simon, consegnando nelle mani di Louise quella fotografia simile «à une feuille morte, avec son assemblée de personnages morts posant» (L'herbe, p. 181), incorpora in multisensoriale meditazione narrativa sul tempo e sulla fatica di «endurer l'Histoire (pas s'y résigner: l'endurer)» (L'herbe, p. 31). Mentre un'agonia incartapecorita si consuma insieme all'estate, i numeri riportati al centro esatto del romanzo sono paragonati a vestigia «qui semblent non pas avoir résisté au temps mais être en quelque sorte le temps lui-même» (L'herbe, p. 97); il muro compatto che quelle cifre costruiscono è paragonato alle inscrizioni latine sui monumenti antichi, «les rangées de mots elles-mêmes comme maçonnées, elles-mêmes semblables à d'indestructibles murailles destinées à durer plus longtemps que le temps même» (L'herbe, p. 105). Non fosse che l'intero edificio comparativo - «fabuleux mausolée fait de temps amoncelé sur un peu de cendre: des cendres, du néant, et, pardessus, un entassement de vide dans ou sur lequel les fantômes des actions accomplies apparaissent avec une désolante insignifiance, concrétisés non par ces ambitieuses inscriptions tracées sur les pierres tombales mais par les dérisoires symboles de l'argent, dans une monnaie dépréciée» (L'herbe, p. 115) - finisce per sgretolarsi al pari della libresca, «insatiable et crédule soif de connaître et de dominer qui est l'expression par l'homme du refus de sa condition» (L'herbe, p. 187).

Dal canto suo, nella suite di frammenti di vita - propria e altrui - che funge da Préface a Les comptes du temps, Pascal Quignard giustappone i carnets di Artémise Simon alle raccolte di ricette di cucina - la «recette (recepta)» essendo «ce qu'on a reçu du temps lui-même» (p. 38) - e ai Festins imaginaires sognati dagli affamati nei campi di concentramento e pubblicati da Anne Georget, agli elenchi di agenda e memoranda e ai gribouillis informi di suo nonno - lo storico della lingua Charles Bruneau - e di sua madre, entrambi colti da demenza senile, per concludere sul ritorno infranto dai campi di Bruno Bettelheim. Illustra così il «fonctionnement magique» (p. 41) della lista in quanto argine che il gesto, la «motion» (p. 40) di scrivere erge per resistere e endurer, funzionamento riassunto nel binomio che dà il titolo alla prefazione: Écrire, tenir (pp. 37-47).

5 Ora, se Mireille Calle-Gruber, in merito agli archivi lasciati da Claude Simon in eredità ai suoi lettori e studiosi presso la Bibliothèque littéraire Jacques Doucet, insiste sul recevoir per a propria volta dare «don et abandon absolus» (p. 15), del gesto di “abban- 
donare" ella declina le implicazioni - donazione e offrande, lascito e adresse, envoi, ma anche muta, metamorfosi e morfogenesi: l'archivio come custodia, nonché riserva, serbatoio - nelle vesti di curatrice di Sur le geste de l'abandon di Pascal Quignard (Paris, Hermann, 2020). Il volume è stato pubblicato in occasione dell'esposizione delle carte $\mathrm{e}$ dei disegni recentemente depositati dall'autore presso la Bibliothèque Nationale de France, rappresentata nel libro dagli interventi della Presidente Laurence Engel (Faites entrer l'infini, pp.3-4) e dal direttore delle collezioni manoscritte Olivier Wagner (Fragments d'une écriture, pp. 5-8).

Il catalogo contiene quanto è sfuggito ai rituali feux de joie, insieme sacrificali e rigeneranti, di uno scrittore che a lungo ha distrutto gli avantesti delle proprie opere: è la «scène du feu» «Où dansent les cendres de tous les manuscrits des livres publiés dans l'année» (Une journée de bonheur, cit. a p. 20). Si tratta dunque in buona parte di carte e immagini relative a testi recenti, a progetti in corso o abbandonati, come la maquette del De taciturnis illustrata da Pierre Frilay: concepito tra il '76 e il '77, il libro non è stato realizzato a causa dell'improvvisa morte dell'artista, mentre il testo è confluito nei Petits traités. Spiccano i dossiers di Tous les matins du monde e, soprattutto, di Terrasse à Rome, ossia i monocromi "Meaumes", dal nome dell'incisore del Seicento protagonista di quest'ultimo romanzo e controfigura di Ludwig von Siegen, l'inventore della manière noire prediletta da Quignard nella misura in cui, cinerea, l'immagine sembra sorgere dall'oscurità originaria. A Ovidio, ad Apuleio sono ispirati altri disegni. I riflessi delle pitture parietali di Lascaux e la sagoma del tuffatore di Paestum accompagnano l'elaborazione di Boutès. Sommate alle numerosissime immagini mobilitate nell'opera di P. Quignard, quelle esposte e ora custodite alla BNF formano una sorta di galleria intima, cui si aggiungono quelle del Fauno Barberini, paragonato all'estasi di Santa Teresa del Bernini nel corso della conférence-performance tenuta dall'autore in occasione della mostra e visionabile sul sito della Biblioteca (Sur le geste perdu de l'abandon, pp. 147-168), oppure La femme à la souricière di Claude Mellan, derivata dalla tela di cui nel Commentaire sur la Hersé de Poussin (pp. 169-184).

7 Se nei testi qui riuniti, lo scrittore ricapitola il proprio pensare l'immagine - arcaica, onirica, fantomatica, l'immagine "vede assente", scaturisce dall'invisibile cui rinvia - e amplia il proprio discorso sulla lettera come immagine, i suoi disegni evidenziano come e quanto, a monte della scrittura, l'immagine "pensi". Essa condensa, tematizza e proietta un desiderio in margine alla scrittura a venire, in divenire (Images pour écrire, pp. 37-40): «griffonnage qui n'est pas encore une écriture articulée, syntaxée. Elle est indissociable des premiers mots du manuscrit. Elle cherche» (p. 39), afferma l'autore. Nel suo insieme, nota M. Calle-Gruber in Sur le don (pp. 9-35), questo libro dà a vedere la mano che disegna e scrive e la mano che dà, dona: la mano che cerca, che pensa. 